
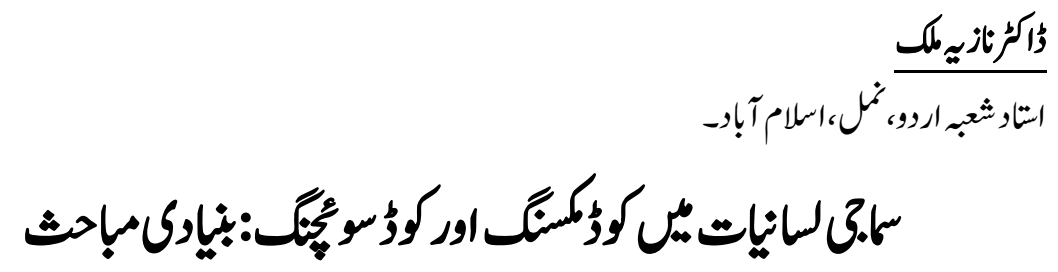

Dr. Nazia Malik

Assistant Professor, Department of Urdu, NUML, Islamabad

\title{
Code Mixing and Code Switching in Sociolinguistics: Basic Discussions
}

The scientific study of language is called linguistics. Literature has a deep connection with linguistics. Language is a social function which is why it is called a branch of linguistics, sociolinguistics. The study of language in the context of sociolinguistics in context. Sociolinguistics actually depends on multilingual and multilingual topics and our country Pakistan is a multilingual country in terms of national and regional. People used to mix several languages during the conversation and do code mixing and code switching. In particular, the mixture of English language is considered a source of pride and English language is the religion of colonial societies. Now English language has been absorbed into Urdu language and has become a part of it. Code mixing and code switching is present in every society and language of the world. Due to this, languages are moving towards progress and decline. Written to explain Western techniques.

Keywords: Sociolinguistics, Code Mixing, Code Switching, Colonial, Bilingual, Multilingual, English Language, Intervention, Mixing, Western Term, External Sentence, Inner Sentence.

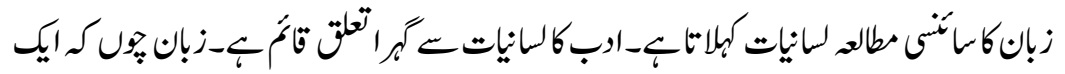

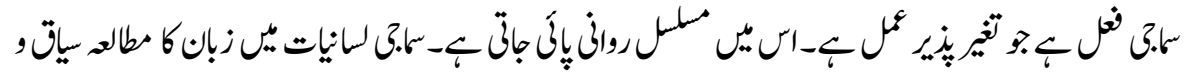

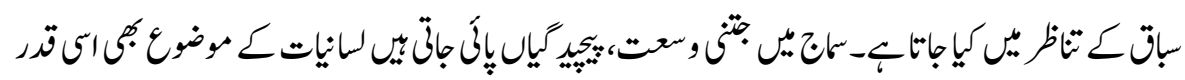

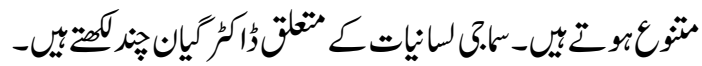


ISSN (Print): 2709-9636 | ISSN (Online): 2709-9644

Volume 2, 2021(Issue III, July to September)

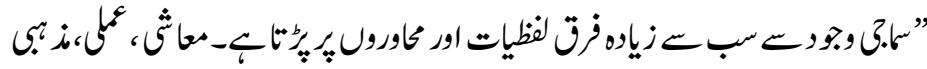

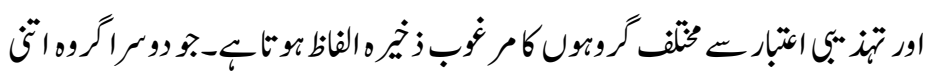

$$
\text { (1)، كثت }
$$

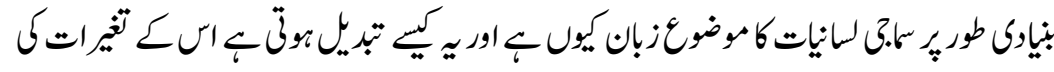

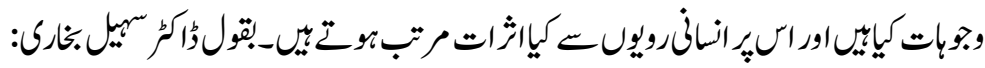

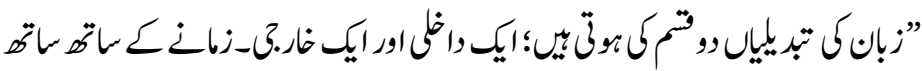

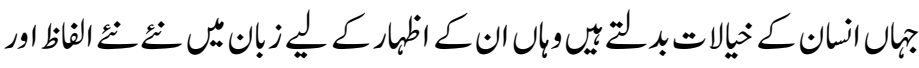

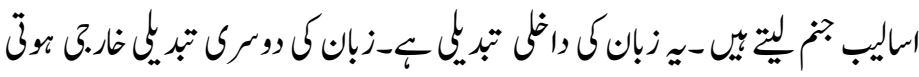

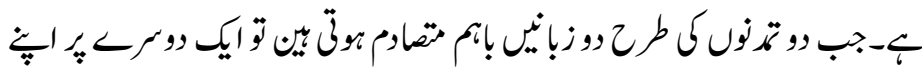

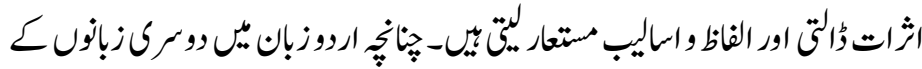

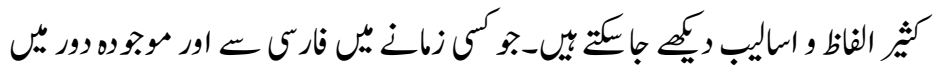

$$
\text { انتريزكس. }
$$

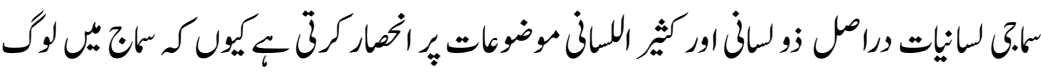

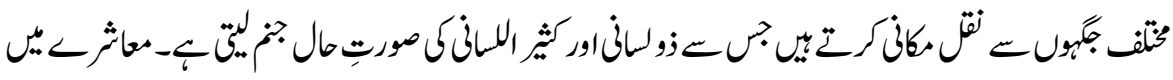

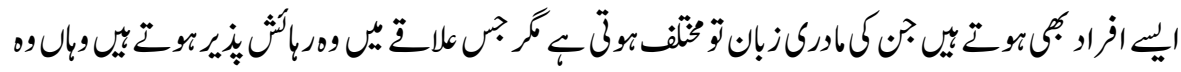

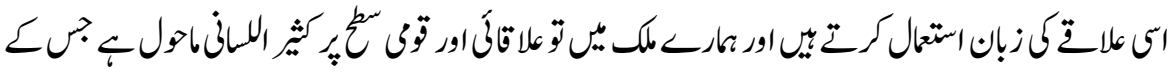

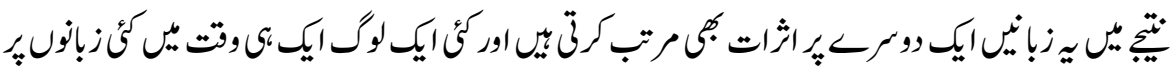

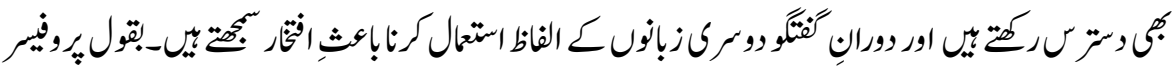

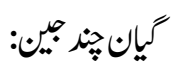

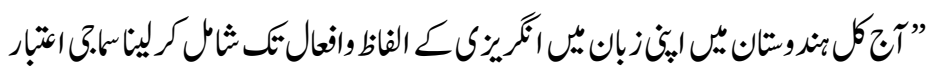

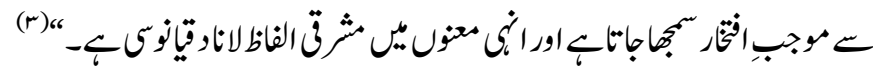


ISSN (Print): 2709-9636 | ISSN (Online): 2709-9644

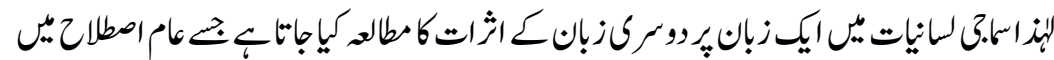

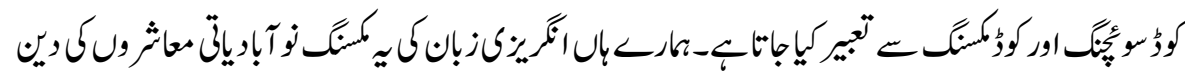

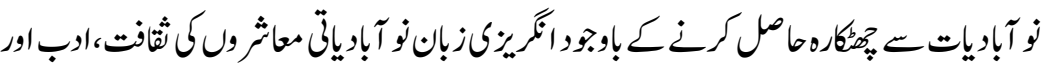

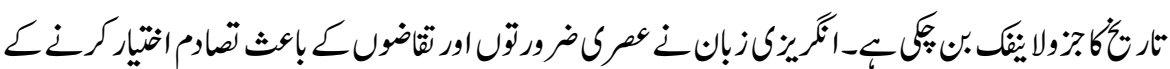

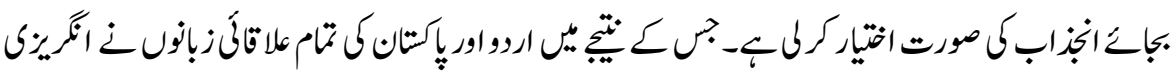

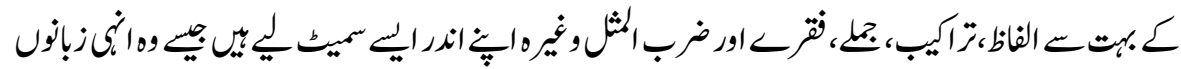

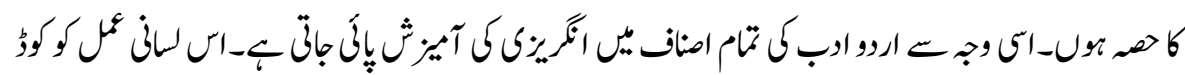

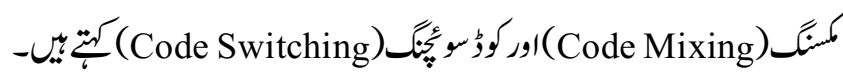

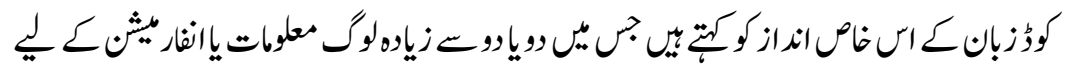

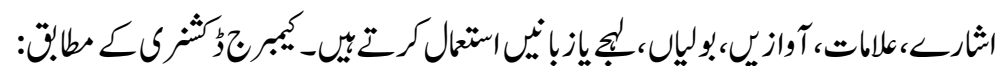

"Code noun (Language) a language or dialect (a form of a language that people speak in a particular part of a country)

(People often switch codes depending on the conversational context)."(4)

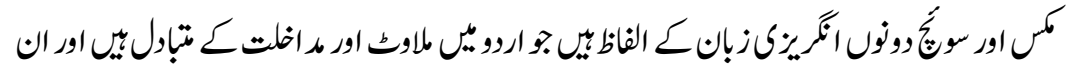

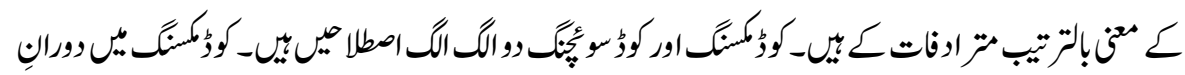

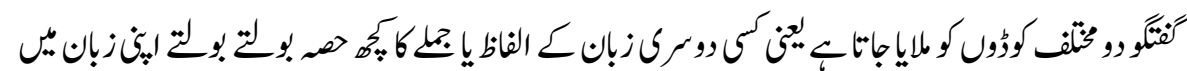

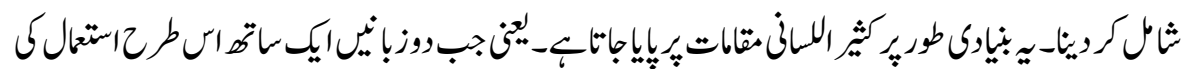

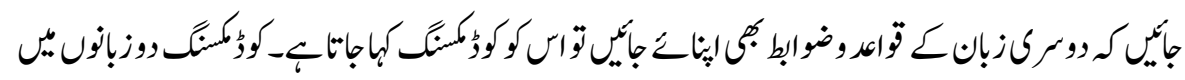

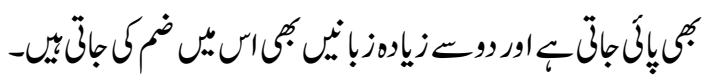

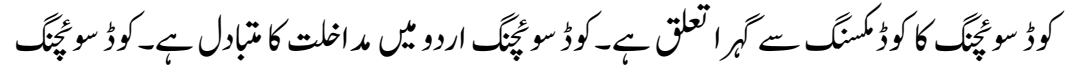

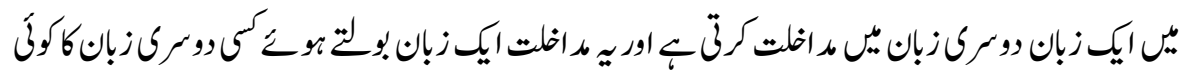


ISSN (Print): 2709-9636 | ISSN (Online): 2709-9644

Volume 2, 2021(Issue III, July to September)

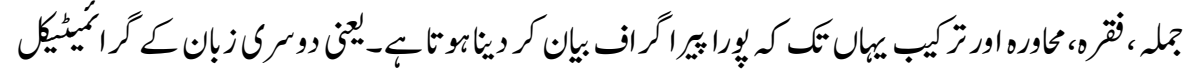

ؤما غ

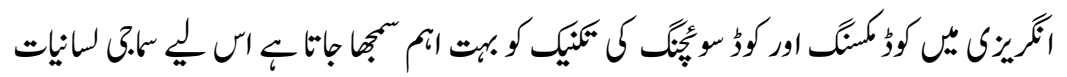

Socio Linguistics

"Code switching process of shifting from one linguistics code (a language or dialect) to another depending on the social context or conversational setting."

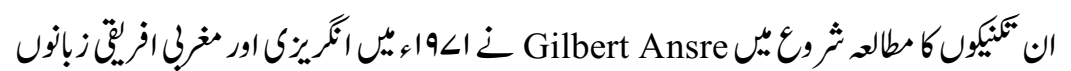

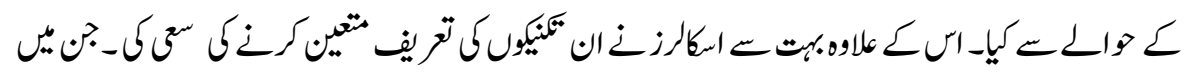

Atoye1994 ‘Hymes1974 ‘Akere1977،Bokamba 1989،Kachru 1989

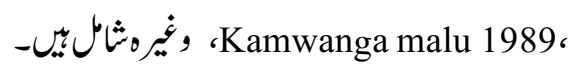

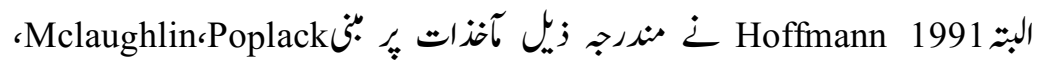

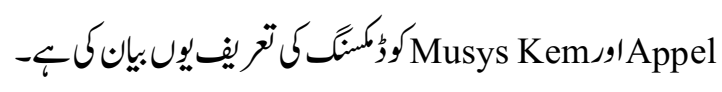

"Switches occurring at lexical levels within a sentence (Intra-sentential switch) are referred to as "Code Mixes" and "Code Mixing”, on the other hand, changes over phrases or sentences (Inter-sentential) including tags and excavation at either end of the sentence, are called code switches and code switching."

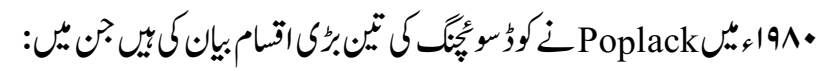

$$
\begin{aligned}
& \text { Tag Switching - - }
\end{aligned}
$$

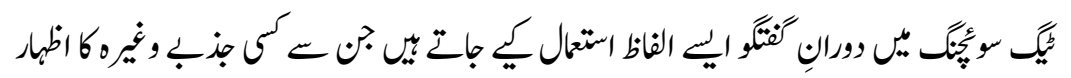

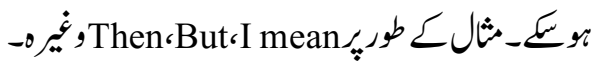




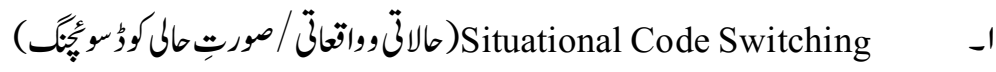

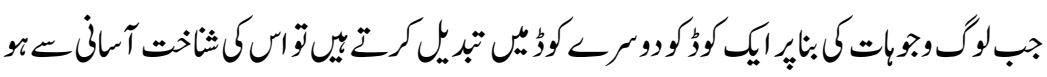

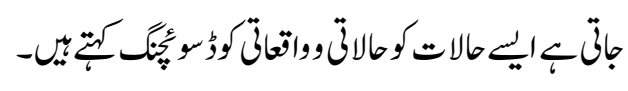

Metaphorical Code Switching

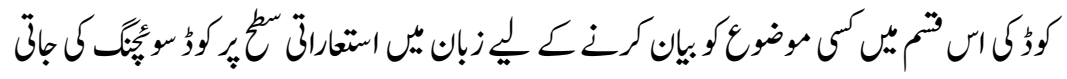

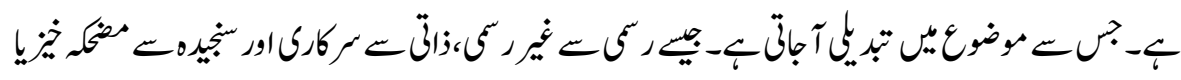

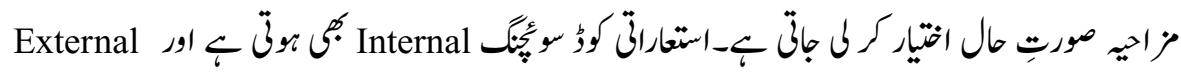

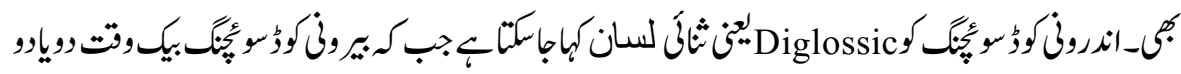

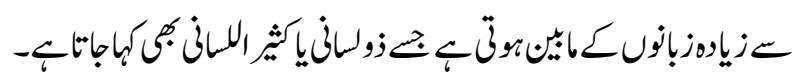


ISSN (Print): 2709-9636 | ISSN (Online): 2709-9644

Volume 2, 2021(Issue III, July to September)

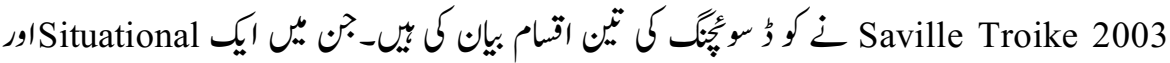

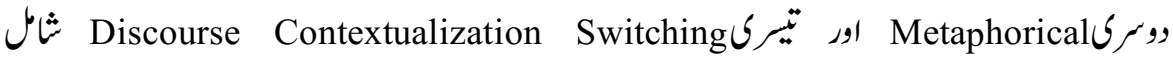

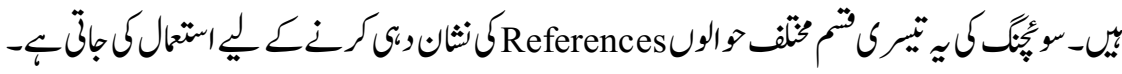

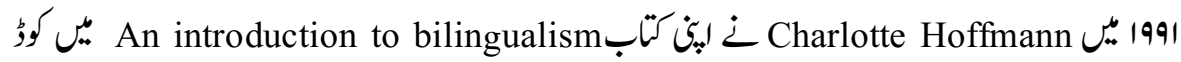

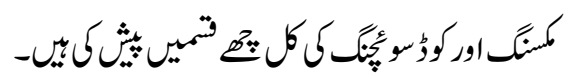

إن Inter Sentential

(اندروظجمل)Intra Sentential

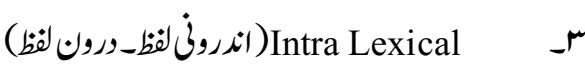

Establishing Continuity with the previous speaker

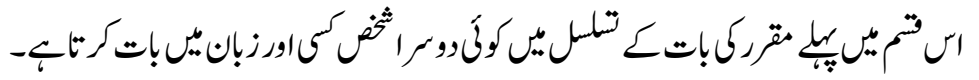

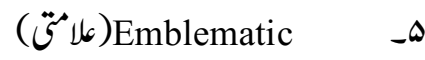

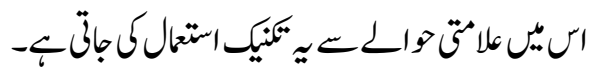

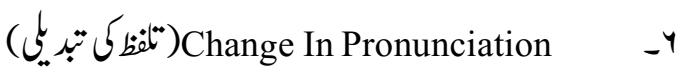

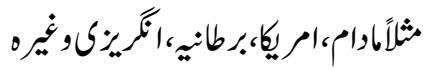

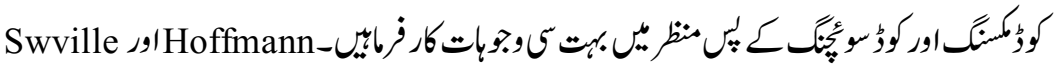

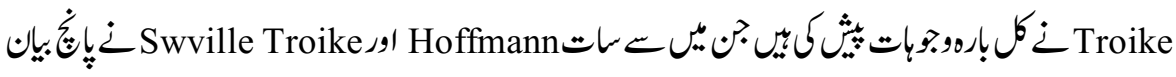

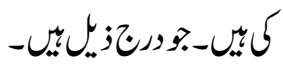

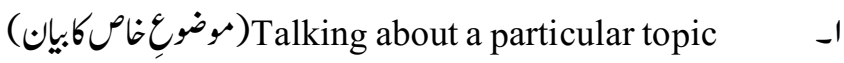

Quoting somebody else -

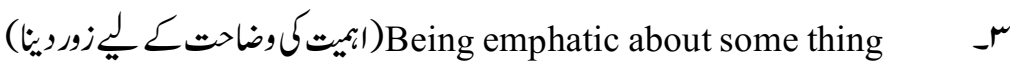
( هـ 
ISSN (Print): 2709-9636 | ISSN (Online): 2709-9644

Expressing group identity

To soften or strengthen request or command

$-\wedge$

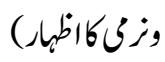

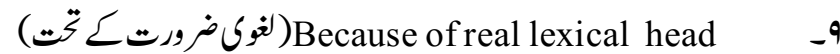

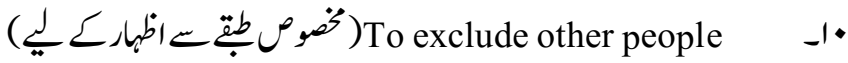

(م) Humorous effects

Personal style

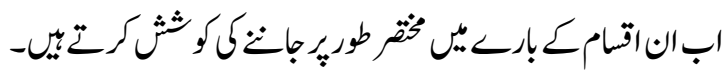

Talking about a particular topic

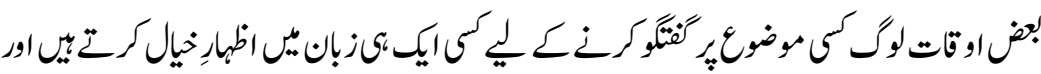

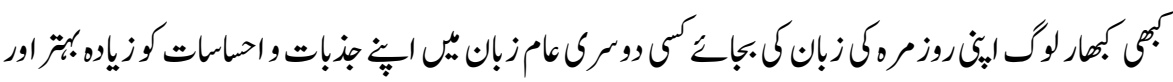

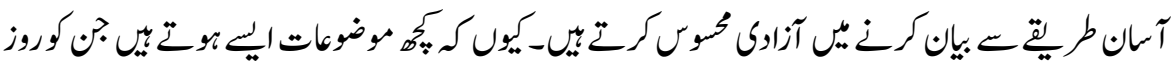

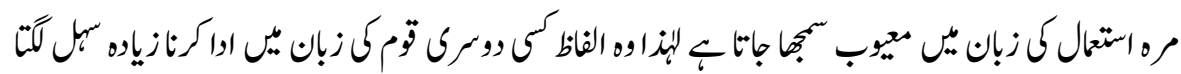

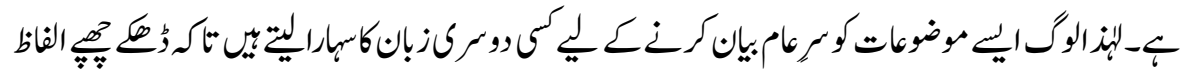

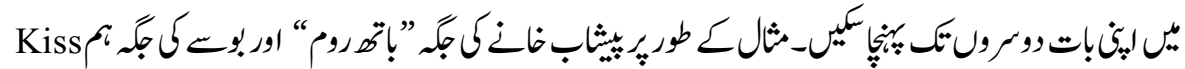

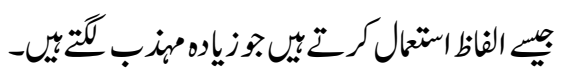

Quoting somebody else

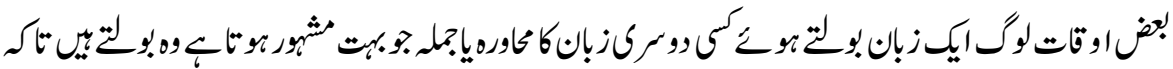

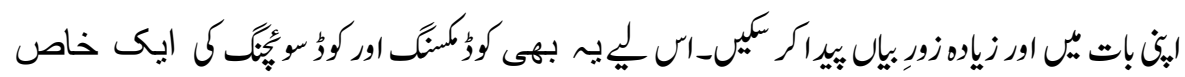

Being emphatic about some thing 
ISSN (Print): 2709-9636 | ISSN (Online): 2709-9644

Volume 2, 2021(Issue III, July to September)

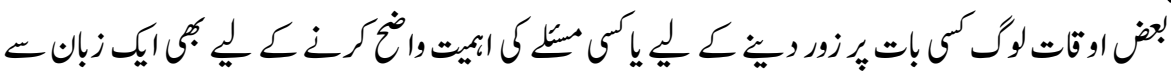

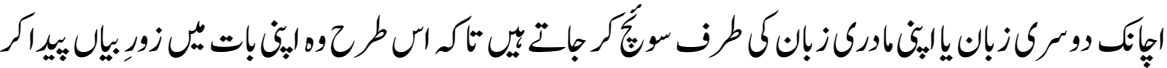

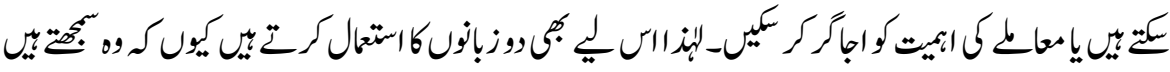

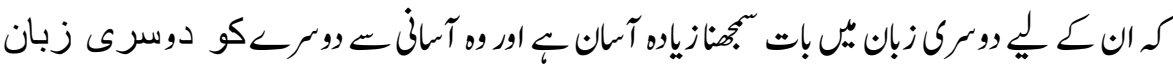

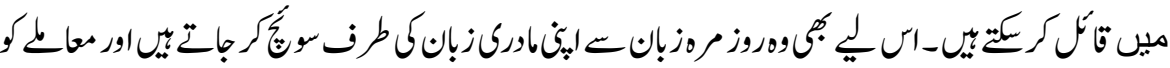

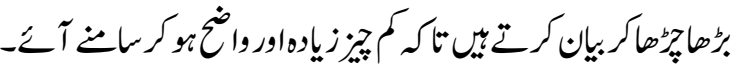

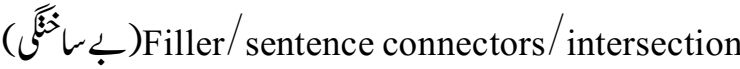

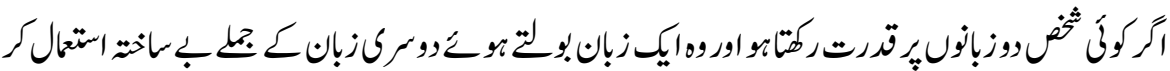

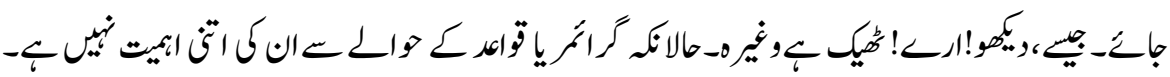

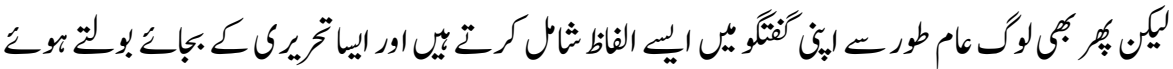

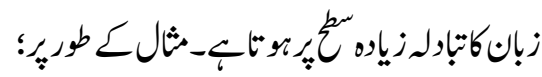

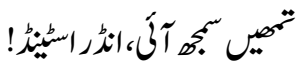

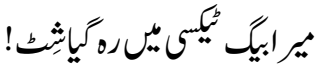

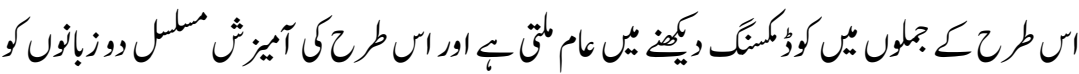

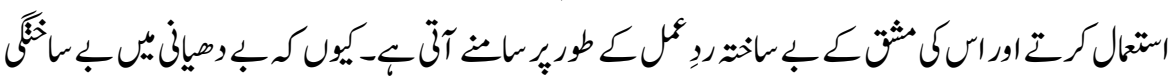

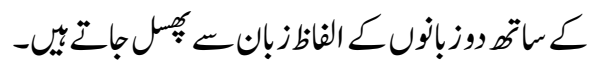

Repetition used for clarification

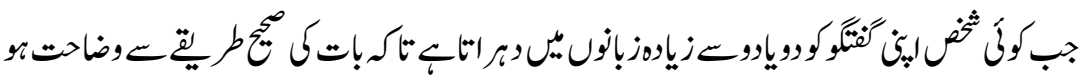

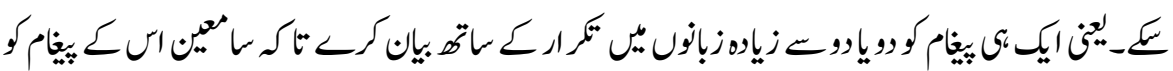

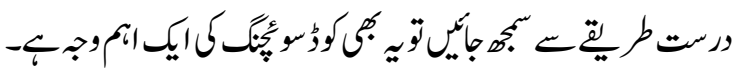
Intention of clarifying speech content 


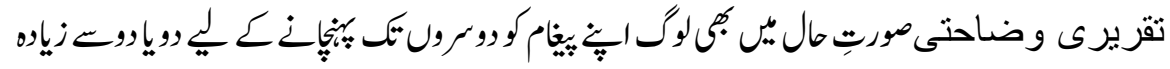

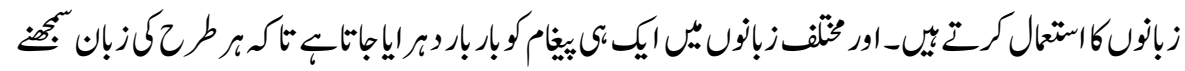

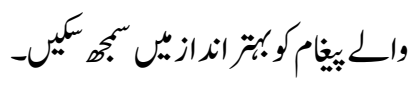

Expressing group identity

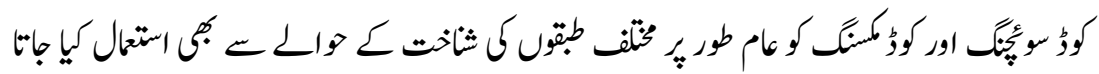

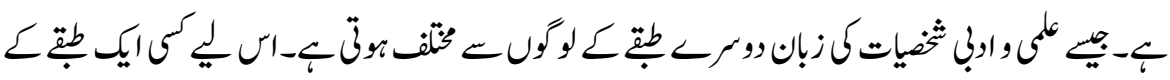

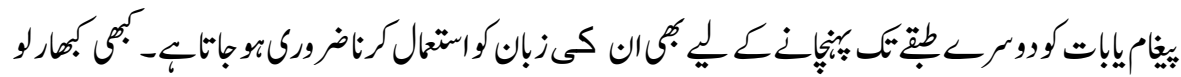

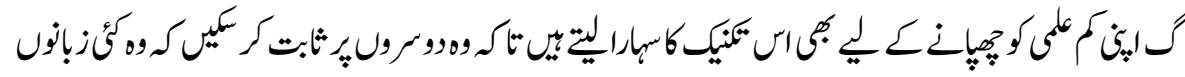

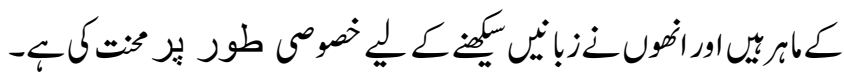
To soften or strengthen request or command

$$
\text { (2) }
$$

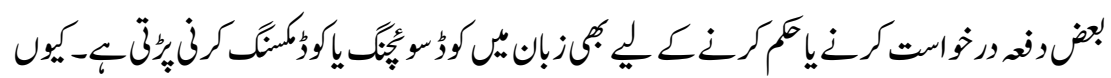

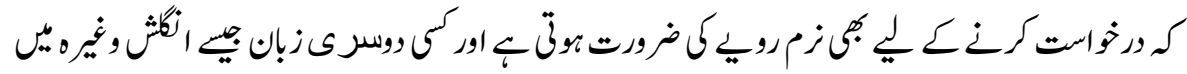

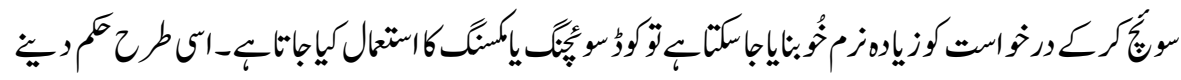

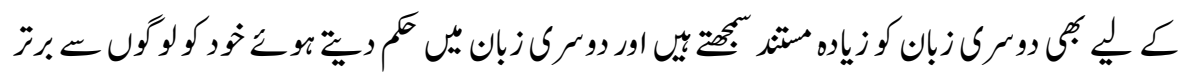

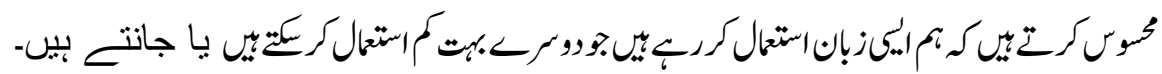

$$
\text { Because of real lexical head }
$$

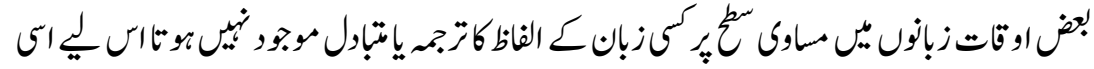

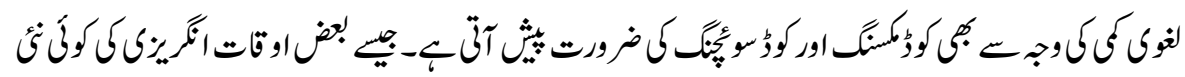

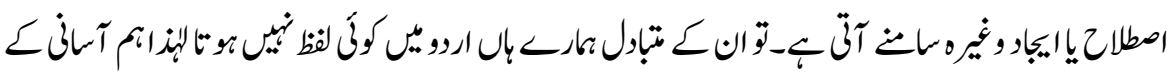

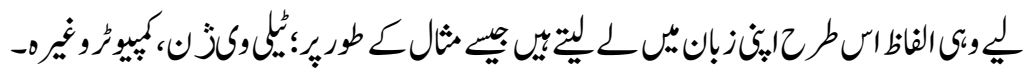

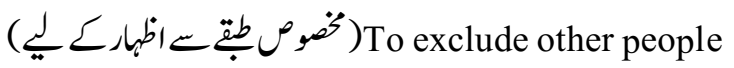




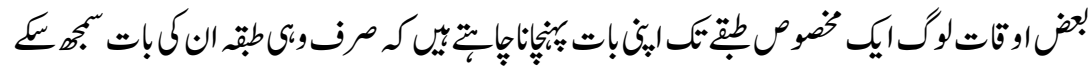

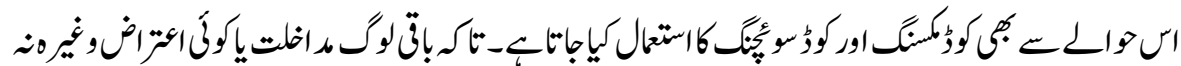

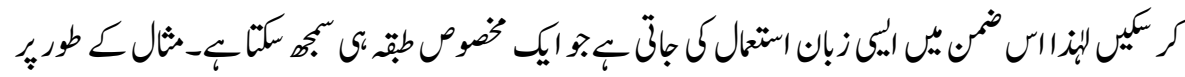

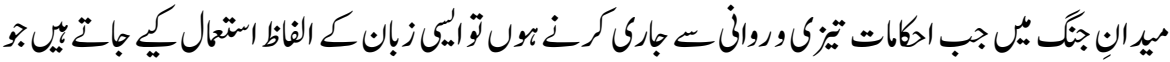

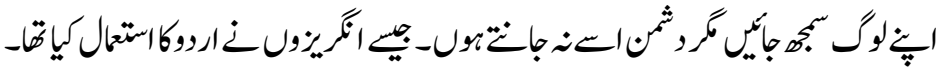
(م)Humorous effects

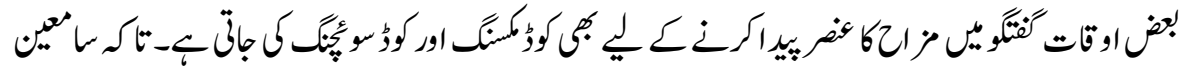

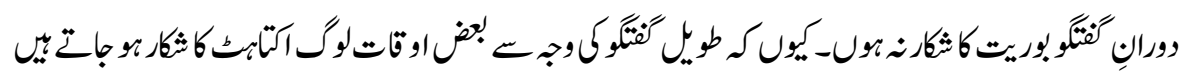

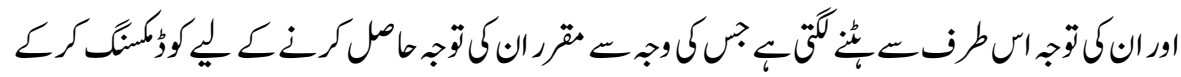

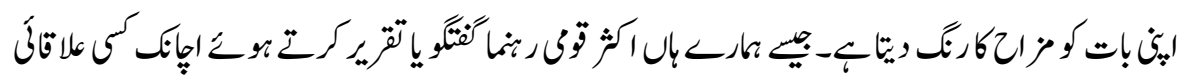

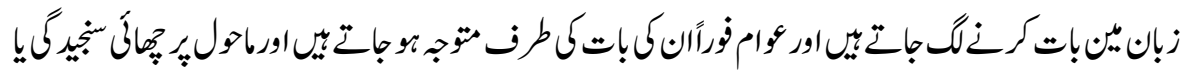

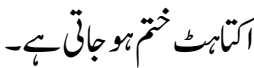

\section{Personal style}

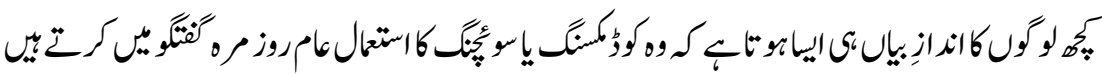

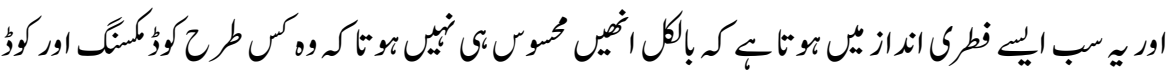

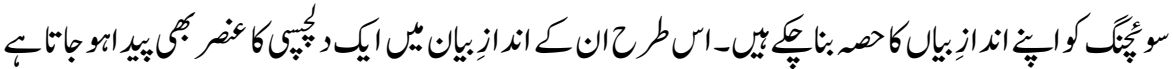

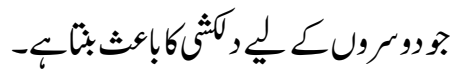

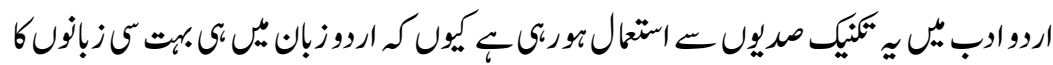

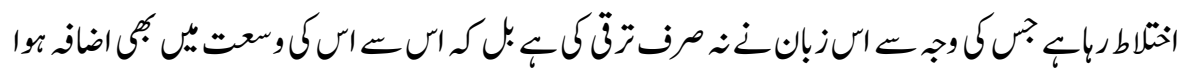

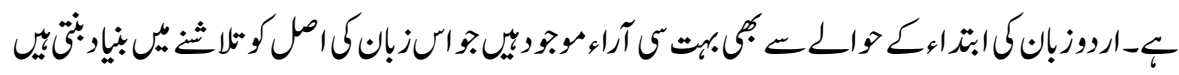

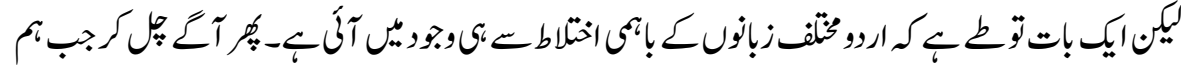

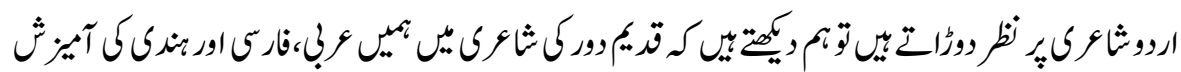

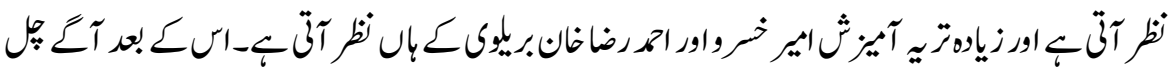




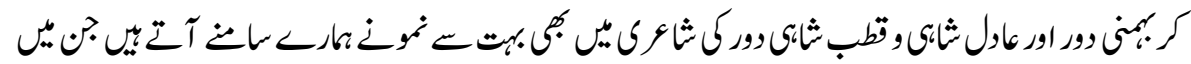

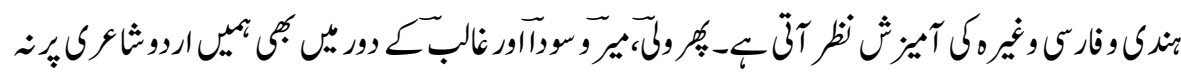

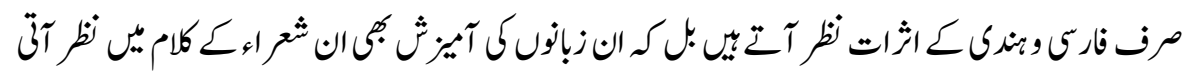

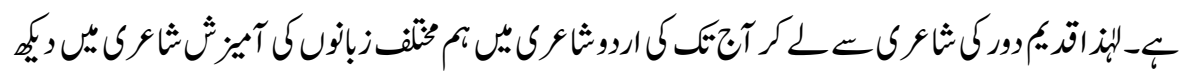
كتم: بـ

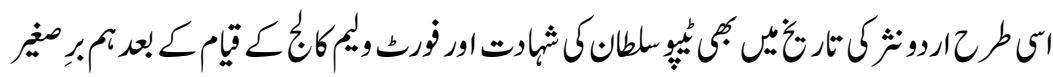

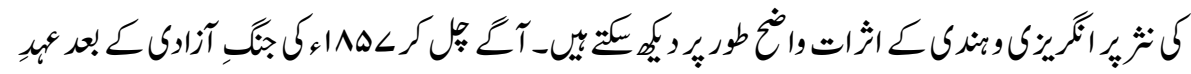

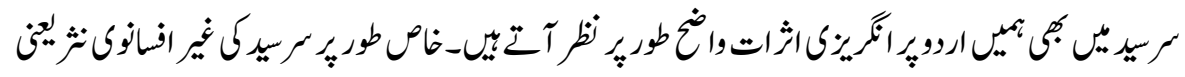

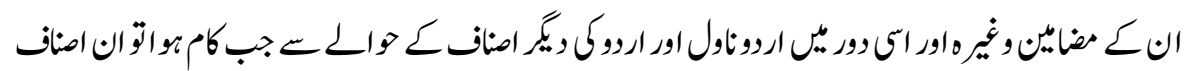

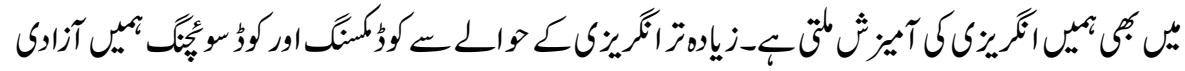

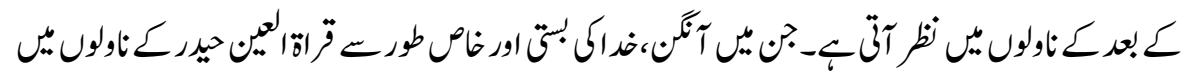

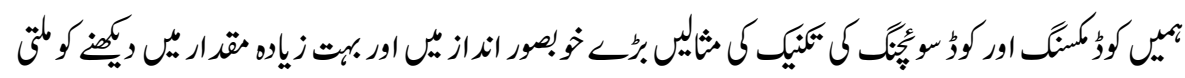

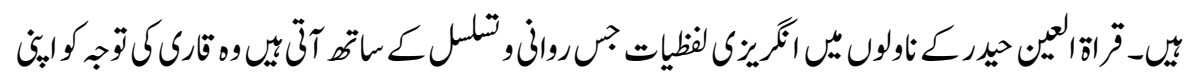

$$
\text { جانب متوج كرق بيل- }
$$

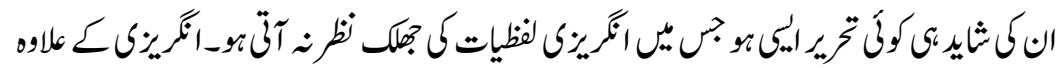

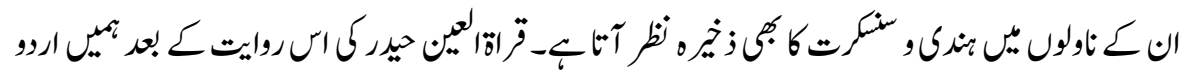

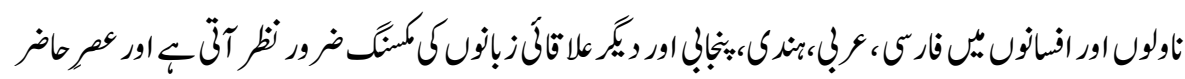

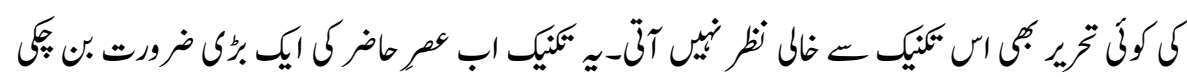

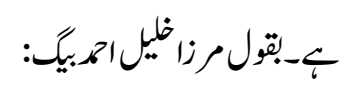

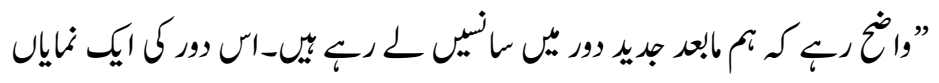

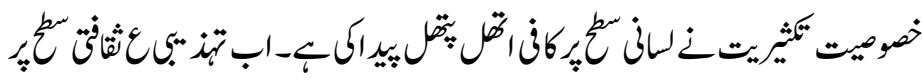

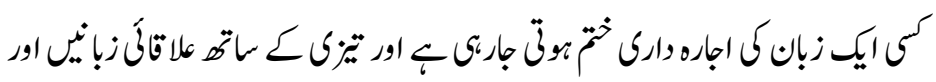


ISSN (Print): 2709-9636 | ISSN (Online): 2709-9644

Volume 2, 2021(Issue III, July to September)

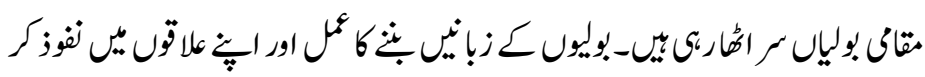

$$
\begin{aligned}
& \text { (L)، بإسن }
\end{aligned}
$$

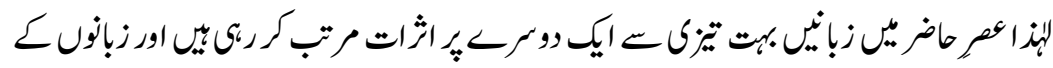

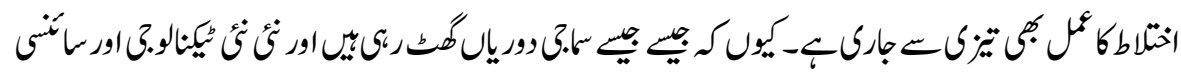

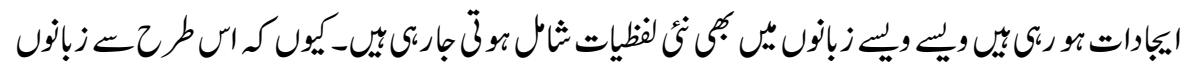

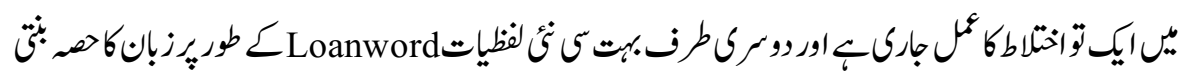

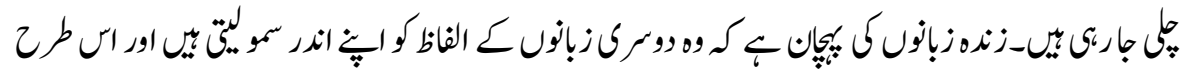

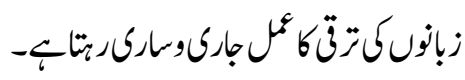

$$
\text { حوالد جات }
$$

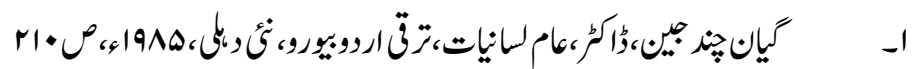

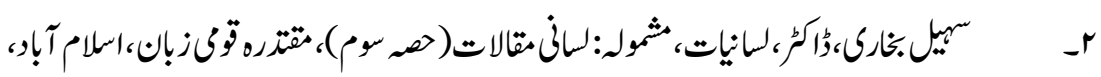

$$
\operatorname{Lr} \rho_{91991}
$$

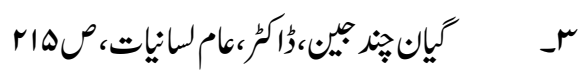

https://www.britannica.com/topic/code-mixing - https://www.britannica.com/topic/code-switching - - Haffmann, c, An Introduction to bilingualism, P:104 - - -

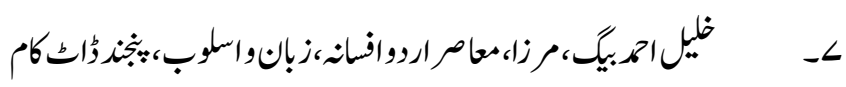

